\title{
TOMOGRAPHY OF HUMAN BODY USING EXACT SIMULTANEOUS ITERATIVE RECONSTRUCTION ALGORITHM
}

\author{
Sayantika Saha ${ }^{1}$, Gargi $\mathrm{Pal}^{2}$, Subarna Pyne $^{3}$ and Sudip Mandal ${ }^{4}$ \\ 1,2,3,4 Department of Electronics and Communication Engineering, \\ Global Institute of Management \& Technology, Krishnagar, India. \\ \{ss.comm12, gogosrk799, boniece23, sudip.mandal007\} @gmail.com
}

\begin{abstract}
In this paper an Exact Simultaneous Iterative Reconstruction Algorithm is developed and applied on a large semi human size normal biological model and a diseased model (liver region affected) to verify the efficiency of the algorithm. The algorithm is successfully reconstructed the normal model having 15\%-20\% perturbation i.e. change in permittivity during disease. In diseased case, reconstructed imaginary part of complex permittivity clearly detects the affected zone and it may help the medical diagnosis. Hence it may be a powerful tool for early detection of cancerous tumors as the interrogating wave is a noninvasive one at the ultra high frequency range. The resolution of this system is increased with the reduction of wavelength by immersing the antenna system and the model in saline water region. The advantage of this algorithm is that the calculation of cofactor are done offline to save the computational time and cofactors are expressed as a function of distances irrespective of their positions .
\end{abstract}

\section{KEYWORDS}

Imaging, Reconstruction, Iteration, Complex Permittivity, Inhomogeneities, Perturbation, Exact algorithm.

\section{INTRODUCTION}

Tomography is a imaging technique to reconstruct the image of cross sectional view of human body on the basis of measuring the complex permittivity of each cell. Normal and diseases cells have different permittivity due to different water content in them. By measuring different permittivity of human organs, different tomography technique can be used for medical diagnosis. During the past few years much attention has been drawn for imaging with attractive form of energy such as low level microwaves, ultrasound and magnetic resonance on account of the awareness of ionizing hazards of x-ray computed tomography. Ultrasonic imaging has already found widespread application but high attenuation and dispersion of ultrasound makes it unsuitable for detecting soft tissues like lungs. MRI scan is very much expensive and not fully free of hazards.

On the other hand microwave region of electromagnetic spectra has many advantages when it is used to interrogate the biological media. The dielectric properties of water molecule dominate the interaction of microwaves and biological system. Thus it is possible to reconstruct the image of the biological target by interrogating it with microwaves. The complex permittivity of the tissue

Rupak Bhattacharyya et al. (Eds) : ACER 2013,

pp. 437-443, 2013. (C) CS \& IT-CSCP 2013

DOI : 10.5121/csit.2013.3241 
changes with water content in it and thus makes it possible to discriminate the healthier tissue from the diseased one.

Microwave tomography lacks in resolution due to its large wavelength and when object inhomogeneity is comparable to the wavelength of microwave frequency (preferably less than 1 $\mathrm{GHz}$ ), energy transmission must be discussed in terms of the scattered fields by the inhomogeneities. Hence reconstruction of constitutive parameter distribution of an object is an inverse scattering problem. Attempts have been made to develop different reconstruction algorithms among which Diffraction Tomography using Born \& Rytov approximation [1] is valid when the inhomogenities in the objects is weakly scattering. The Complete First Order Algorithm by Datta and Bondopadhyay using moment method solution [2] [3] depicts the image of small biological model but could not reach the level of satisfaction. Improved $1^{\text {st }}$ Order and $2^{\text {nd }}$ Order Iterative Reconstruction Algorithm developed by Datta and Purkait[4] ans [5], [6], [7] is applicable to large perturbation up to $(20 \%)$ but not for large model due to dominating characteristics of higher order non linear terms.

In this paper an Exact Simultaneous Iterative Reconstruction Algorithm has been developed in section 2, which can be satisfactorily applied to a large semi human size biological normal and diseased model. The rest of the paper is organized as follow, in section 3 cofactor calculations with respect to distances between cells is described. Numerical Model and proposed antenna system is explained in Section 4. Next different cases of reconstruction are described. Results and Figures after simulations are shown in Section 6.Conclusion \& References are given in next two sections respectively.

\section{EXACT SIRT ALGORITHM}

According to Richmond [5], a system of linear equations are obtained by enforcing the condition that total field at the center of each cell must be equal to the sum of the incident and the scattered field. From Richmond's Theory the field distribution in unperturbed homogeneous medium is expressed by equation

$$
[\mathrm{C}] \cdot[\mathrm{E}]=\left[\mathrm{E}^{\mathrm{in}}\right]
$$

where $[C]$ is a $(n \times n)$ coefficient matrix of homogeneous medium, $E_{i}^{i n}$ is the incident field at $i$-th cell in free space and $E_{i}$ is the internal field at $i$-th cell when the medium is assumed to be homogeneous one having known permittivity distribution.

In presence of inhomogeneous biological targets the permittivity values of the cells are perturbed simultaneously by small amounts of $\Delta \varepsilon_{i}(i=1,2, \ldots, n)$ and the corresponding changes in the internal fields are $\Delta E_{i} \mathrm{~s}$ then perturbed field is expressed as

$$
\left[\mathrm{C}^{\prime}\right] \cdot\left[\mathrm{E}^{\prime}\right]=\left[\mathrm{E}^{\mathrm{in}}\right]
$$

Where [ $\left.C^{\prime}\right]$ is the coefficient matrix corresponding to the inhomogeneous medium and $E^{\prime}$ (=E $+\Delta E)$ is the modified field in the $i$ th cell under perturbed condition.

Here an Exact SIRT algorithm has been developed where difference between $E$ and $E^{\prime}$ at a particular cell is expressed in terms of unknown permittivity, relevant cofactors of coefficient matrix corresponding to homogeneous medium and perturbed internal fields.

The incremental field in $i$-th cell is represented by 


$$
\Delta E_{i}=-x_{i} E_{i}^{\prime}+\sum_{j=1}^{N} x_{j} E_{j}^{\prime} \frac{M_{j i}(0)}{\Delta(0)}
$$

where $\Delta(0)$ and $M_{j i}(0)$ are the determinant and cofactor of $(j, i) t h$ element of unperturbed coefficient matrix $[C]$ respectively and $x_{j}=\left(\varepsilon^{\prime}-\varepsilon_{w}\right) /\left(\varepsilon_{w}-1\right)$ is the requisite fractional change of permittivity of $j$ th cell, $\varepsilon_{w}, \varepsilon^{\prime}$ are the complex permittivity of saline water and model cell respectively.

Iteration was initiated with an assumption $\Delta E_{i}=0$, i.e. Internal fields of the unperturbed medium are taken as trial values for perturbed internal field $E_{j}^{\prime}$. until difference between two successive fields is less than $0.1 \%$.

Now, the resultant change in the scattered field at a particular receiver location owing to change of internal fields at the different cells of the medium caused by the perturbation of complex permittivity distribution is given by

$$
\Delta \mathrm{E}_{\mathrm{R}}=\sum_{\mathrm{j}=1}^{\mathrm{N}} \mathrm{x}_{\mathrm{j}} \mathrm{E}_{\mathrm{j}}^{\prime} \frac{\mathrm{M}_{\mathrm{j}, \mathrm{R}}(0)}{\Delta(0)}
$$

Therefore, for $k$ th beam the difference in field equation at the $l$ - th receiver

$$
E_{R m l}(k)-E_{R o l}(k)=\sum_{j=1}^{N} \Delta x_{j} E_{j}^{\prime} \frac{M_{j R}(0)}{\Delta(0)}+\sum_{j=1}^{N} x_{0 j} E_{j}^{\prime} \frac{M_{j R}(0)}{\Delta(0)}
$$

Where $E_{R m l}(k)$ and $E_{R o l}(k)$ denotes the scattered field at the $l$ th receiver location for the $k$ th beam in the inhomogeneous and homogeneous numerical model respectively. Where $x_{o j}$ is the initial shift in requisite fractional change in permittivity in $j$ th cell from saline water. Because of the limitation of averaging procedure the initial values of complex homogeneities of different region are assumed to be deviated from the model value by less than $20 \%$.

\section{Cofactor Calculation}

Since the number of operations per calculation is proportional to $N^{3}$, where $N$ is the number of cells for which the complex permittivity have to be reconstructed. Hence, individual computation of all co-factors will time consuming and expensive for a model of large number of cells. Instead of computing all the co-factors individually, these are determined as a function of distance between the cells under observation which are free from boundary effect i.e. well within the medium.

\section{Model And Antenna System}

A semi human-size model of 360 cells having complex permittivity identical to those observed in different human organs i.e. liver (46-j10), bone (8-j1.2), pancreas (65-j30), stomach (60-j18), spleen (58-j28), artery (55-j28), fat (25-j5), muscle $(50-\mathrm{j} 23)$ and muscle type $(35-\mathrm{j} 15)$ etc. , surrounded by saline water region having 340 cells, each of 1 sq. $\mathrm{cm}$ in area.

This model is illuminated by a $15 \times 15$ quarter wave dipole array antenna with beam width of 5-6 degree, operating at $480 \mathrm{MHz} 20$ half wave dipoles are used as receiver.

The model and the antenna system are immersed in saline water. This reduces the wavelength from $62.5 \mathrm{~cm}$ (i.e. free space wavelength at $480 \mathrm{MHz}$ ) to $7.16 \mathrm{~cm}$ in the medium due to large 
dielectric constant of water (i.e. 76) which in term increases the resolution by the same factor. To obtain a better focusing control, amplitude and phase conjugations are obtained by placing the array antenna on a spherical surface with focal length of $50 \mathrm{~cm}$.

\section{NUMERICAL SimUlation}

In this section the results on two different cases are simulated for the testing the validity of the proposed algorithm.

\subsection{Case-1:}

This model is assumed containing the exact value of permittivity of normal organ cells.

If there are $k=1,2, \ldots q$ number of beams passing through a particular cell, then from a set of $\mathrm{q}$ number of equations, the values of $\left(\Delta x_{j}\right)$ can be calculated by SIRT technique[6] using equation (5). The same procedure is adopted for all cells one by one assuming the basic concept that all the cells are equally responsible for a change in the receiving field. The iteration process was started with $x_{o j}$ and the internal field corresponding to this initial shift to obtain $\Delta x_{j}$ using equation (5). Each time the field is modified by $\left(x_{o j}+\Delta x_{j}\right)$ with the help of equation (3) until the minimum value of rms deviations [6] of the theoretical output field from the estimated one corresponding to different rays was obtained.Here the image of the model is reconstructed by considering all the rays passing through the cells for 21 different positions. The reconstructed values of complex permittivity in different cells are given by $\varepsilon_{i}^{\prime}=x_{i}\left(\varepsilon_{w}-1\right)+\varepsilon_{w}$ where $x_{i}$ is the reconstructed value of permittivity of $i$-th cell.

The coloured picture of real and imaginary values of complex permittivity in actual and reconstructed normal model are depicted in Fig. 2, Fig. 3respectively using gradation scale where each region is characterized by different colour which is shown in Fig. 1

\subsection{Case-2}

In this case, the model is same as earlier one instead of liver region which is assumed to be affected by some disease. Hence the value of $\varepsilon_{i}^{\prime}$ for liver is changed from (46-j10) to (42-j8). The model is iteratively reconstructed again using same $x_{o j}$ and same iteration process.

The actual and reconstructed real and imaginary part of permittivity in each cell is depicted in Fig. 4 and Fig. 5 respectively.

\section{RESUlTS AND FiguRES}

Using the reconstruction algorithm for all above cases, the experimental data are simulated and the corresponding images are shown below. 


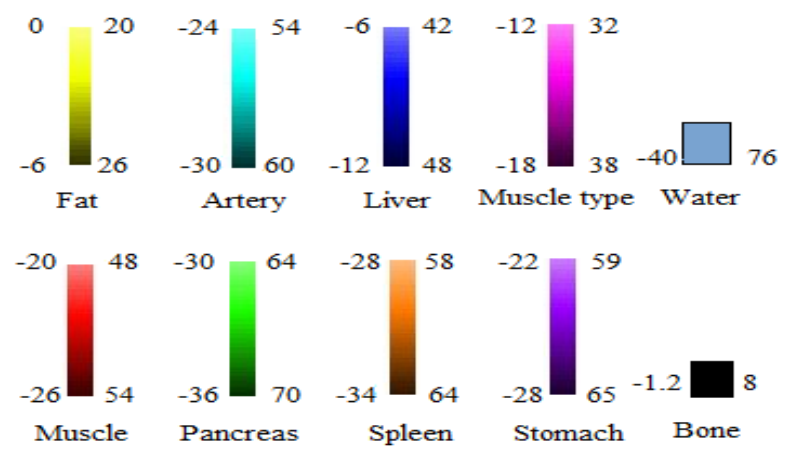

Figure 1: Gradation scale used for the imaging

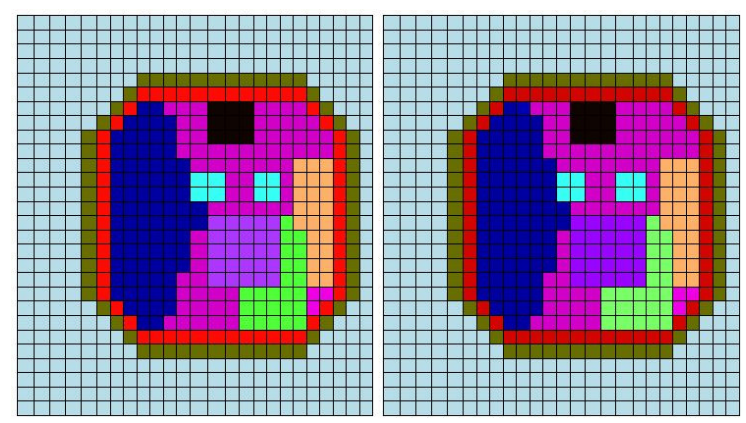

Figure 2: Real and imaginary values of permittivity for normal model respectively

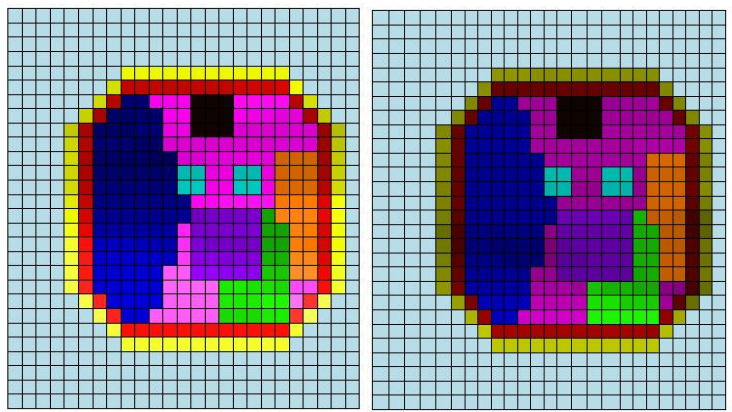

Figure 3: Reconstructed real \& imaginary values of permittivity for normal model respectively

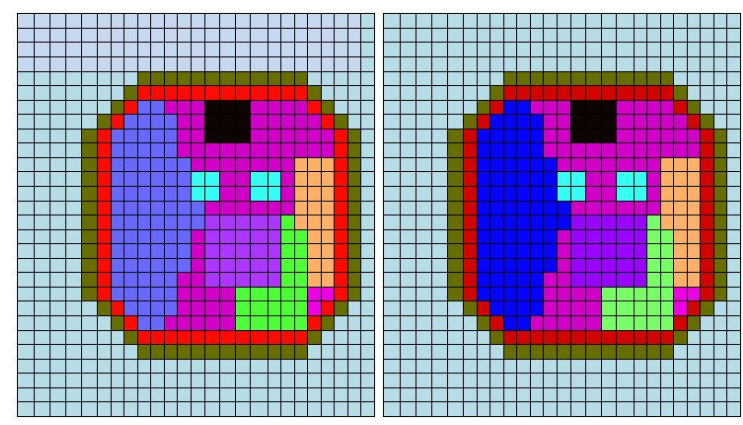

Figure 4: Real \& imaginary values of diseased model respectively 


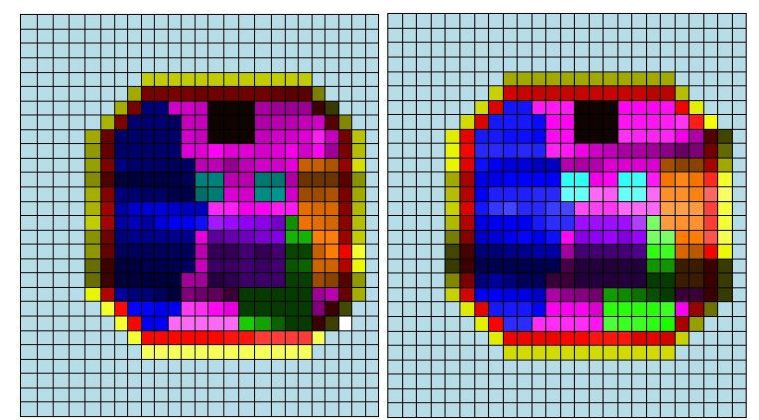

Figure 5: Reconstructed real \& imaginary value of diseased model respectively.

\section{DISCUSSION}

So in this paper, Exact Simultaneous Iterative Reconstruction Algorithm is developed and validated on a large semi human size normal biological model and a diseased model (liver region affected).

Exact Reconstructed images of the normal model are quite satisfactory with clinically acceptable accuracy. But in case of diseased model, the reconstructed image is not as good as expected due to its large perturbation since the diseased liver (42-j8) is perturbed by more than $20 \%$ from the initial trial value (52-j12). The imaginary part of the reconstructed permittivity of the liver region shows that the liver region is affected. The reconstructed complex permittivity in some region deviates from their actual value due to the averaging effect of SIRT method itself.

The offline cofactor calculation can be applied for even larger model efficiently and also reduces the computation time and algorithm complexity is also less. So, proposed tomography technique using exact algorithm can be used for medical diagnosis effectively such as cancer detection etc. of human body.

\section{REFERENCES}

[1] Carosi S, Gragani G L, Pastriorino M \& Sartore M, IEEE, proc H ,Microwave and Antenna Propagation, vol. 141,1994, pp.-445

[2] A. N. Datta \& B. Bandyopadhyay, “ A Simplified Algorithm for Microwave Tomography” IEEE ,Int J Electron, Vol. 141,1985,pp.831

[3] A. Boag, R. Mittra, IEEE Trans on BME, Vol. 42, No.3, 1994, pp 366-372

[4] K. Purkait \& A.N. Datta," An Improved form of Iterative Algorithm for First Order and Second Order Microwave Image Reconstruction" Indian Journal of Pure \& Applied Physics, CSIR,Vol.34,June 1996,pp,420-424

[5] Richmond J H, "Scattering by a Dielectric Cylinder of Arbitrary Cross Section Shape" IEEE, Trans Antenna Propagation, 1965, pp. 334-341

[6] A. N. Datta \& B. Bandyopadhyay, "An Improved SIRT-Style Reconstruction Algorithm for Microwave Tomography", IEEE Trans on BME, VOL. BME-32, NO. 9, September 1985, pp.719722.

[7] K. Purkait \& S. Mandal, "Multiview Microwave Tomography of Biological Body", RTCSP National Conferrance, Electron, Vol-3, March -2011. 


\section{AUTHORS}

Sayantika Saha is pursuing B.Tech degree in Electronics and Communication Engineering from the Global Institute of Management \& Technology. Her research of interest includes Tomography and Microwave.

Gargi pal is pursuing B.Tech degree in Electronics and Communication Engineering from the Global Institute of Management \& Technology. Her research of interest includes Tomography and Microwave.

Subarna Pyne is pursuing B.Tech degree in Electronics and Communication Engineering from the Global Institute of Management \& Technology. Her research of interest includes Tomography and Microwave.

Sudip Mandal received the B.Tech. and M.Tech. Degrees in Electronics and Communication Engineering from Kalyani Govt of Engineering College in 2009 2011, respectively. Recently he has held the position of Head of the Department of ECE in Global Institute of Management \& Technology, Krishnagar and is pursuing $\mathrm{PhD}$ degree from University of Calcutta. His current work includes microwave, tomography, Bioinformatics and Soft computing

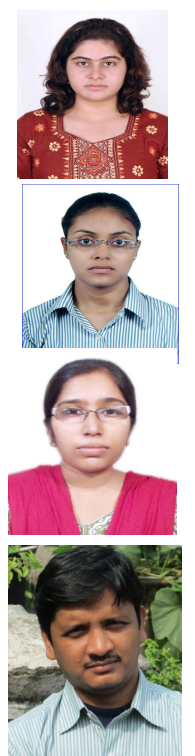

